\title{
ON THE ZEEMAN COMPARISON THEOREM FOR THE HOMOLOGY OF QUASI- NILPOTENT FIBRATIONS
}

Journal Article

Author(s):

Hilton, Peter; Roitberg, Joseph

Publication date:

1976

Permanent link:

https://doi.org/10.3929/ethz-b-000423106

Rights / license:

In Copyright - Non-Commercial Use Permitted

Originally published in:

The Quarterly Journal of Mathematics 27(4), https://doi.org/10.1093/qmath/27.4.433 


\author{
By PETER HILTON and JOSEPH ROITBERG
}

[Received 13 October 1975]

\title{
0. Introduction
}

THIs note constitutes a generalization of the Zeeman comparison theorem for spectral sequences [9]. Zeeman's theorem was based on hypotheses valid for the homology spectral sequence of a fibration with simplyconnected base space; however, his hypotheses were stated purely algebraically and there was no assumption that the spectral sequence was derived from a filtered chain complex-merely that it was a first quadrant sequence and the differentials had the usual bidegrees. Thus Zeeman's version was more general than Moore's earlier comparison theorem [5]; it was also more general in that isomorphism assumptions were only made up to certain dimensions (and so only deduced up to certain dimensions).

We generalize Zeeman's theorem in two directions. The most important direction is that we cover the situation of a quasi-nilpotent fibration; this is a fibration

$$
F \rightarrow E \rightarrow B
$$

in which all spaces are connected, and $\pi_{1} B$ operates nilpotently on $H_{i} F$, $i \geqslant 0$. We say that $(0.1)$ is strongly quasi-nilpotent if it is quasi-nilpotent and if, in addition, $\pi_{1} B$ is nilpotent.

Among the quasi-nilpotent fibrations we find the nilpotent fibrations [2]; these are fibrations $(0.1)$ in which all spaces are connected and $\pi_{1} E$ operates nilpotently [3] on $\pi_{i} F, i \geqslant 1$. If $E, B$ are nilpotent spaces and $F$ is connected then $F$ is nilpotent and $(0.1)$ is a nilpotent fibration, and also strongly quasi-nilpotent. A special case, then, of a nilpotent fibration which is also strongly quasi-nilpotent is

$$
\tilde{X} \rightarrow X \rightarrow K\left(\pi_{1} X, 1\right)
$$

where $X$ is nilpotent and $\tilde{X}$ is the universal cover of $X$. We obtain a very general theorem of Whitehead type by applying our comparison theorem to the homology spectral sequence of (0.2) (see Corollary 3.4); we remark that this spectral sequence violates not only assumption (iii) of [9] but also the refinement of (iii) mentioned on p. 58 of [9].

We should mention at this point that our results strengthen those of Zeeman even when the base is simply-connected-that is, in the case

Quart. J. Math. Offord (2), 27 (1976), 433-444 
Zeeman was considering. For whereas, in Theorem 2 of [9], it was assumed that, in the map of fibrations

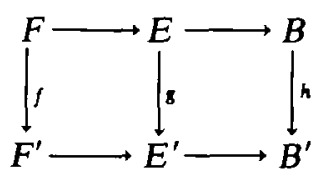

$g$ induces isomorphisms $g_{*}: H_{i} E \rightarrow H_{i} E^{\prime}, i \leqslant N$, and $h$ induces isomorphisms $h_{*}: H_{i} B \rightarrow H_{i} B^{\prime}, i \leqslant P$, in order to deduce that $f$ induces isomorphisms $f_{*}: H_{i} F \rightarrow H_{i} F^{\prime}, i<Q=\min (N, P-1)$ with $f_{*}: H_{Q} F \rightarrow H_{Q} F^{\prime}$ surjective, we obtain (Theorem 3.2) the same conclusion where we weaken the hypotheses by only requiring $g_{*}: H_{N} E \rightarrow H_{N} E^{\prime}$ and $h_{*}: H_{\mathrm{P}} B \rightarrow$ $H_{P} B^{\prime}$ to be surjective instead of isomorphic. This improvement is essential for the deduction of a Whitehead theorem. Similarly, in our versions of Theorems 1 and 3 of [9]-that is, in Theorems 3.1 and 3.5-we make hypotheses which only involve surjection in the top dimensions. These improvements are rendered possible by strengthening the two fundamental lemmas of [9] these improvements appear in $\$ 2$. We remark, with regard to Lemma 2.1 (which improves Lemma 3 of [9], insofar as the latter requires that $\alpha: K \rightarrow K^{\prime}$ be an isomorphism of finitely-filtered abelian grous), that only the improvement in Lemma $2.1(b)$ is exploited in this note. That in Lemma 2.1(a) would come into play if we worked in cohomology rather than homology, as in [6].

The second direction in which we generalize Zeeman's theorem is that we work modulo an acyclic Serre class $C$ of nilpotent groups [4]. (Of course, in the original context of Zeeman's theorem this would amount to working modulo a Serre class of abelian groups in the classical sense [7].) Thus our assumptions and conclusions are all to be understood modulo $C$. We recall here the axioms on a Serre class $C$ of nilpotent groups. Given a non-empty family $C$ of nilpotent groups, we say that $C$ satisfies $(S)$ if, for any central extension of nilpotent groups $N \nrightarrow G \rightarrow Q$,

$$
N, Q \in C \Leftrightarrow G \in C .
$$

We say that $C$ satisfies $(I)$ if

$$
A \in C \Rightarrow A \text { is finitely-generated, }
$$

where $A$ is abelian; and that $C$ satisfies $(I I)$ if

$$
A \in C \Rightarrow \oplus A \in C
$$

where the direct sum is taken over any indexing set. We say that $C$ is acyclic if

$$
A \in C \Rightarrow H_{n} A \in C, \quad n \geqslant 1
$$


Then $C$ is an acyclic Serre class if it is acyclic, satisfies $(S)$, and satisfies $(I)$ or (II). Our theorems apply to maps between any strongly quasinilpotent fibrations (0.1) if $C$ is an acyclic Serre class satisfying (II); and to maps between strongly quasi-nilpotent fibrations with all spaces having finitely-generated homology in all dimensions if $C$ is an acyclic Serre class satisfying $(I)$. It is not hard to show that if $(0.1)$ is a strong quasi-nilpotent fibration in which $F$ and $B$ have finitely-generated homology in all dimensions, then $E$ also has finitely-generated homology in all dimensions. Since all the necessary theory of Serre classes of nilpotent groups was established in [4], there is no difficulty in achieving the desired generalization of the comparison theorem. We will need certain results on homology with nilpotent local coefficients and these are obtained in $\S 1$; the argument, thanks to the results in [4], is no more complicated in the mod $C$ case than in the absolute case $(C=\{1\})$. Corollary 1.4(ii), in the absolute case, was first noted by Dror.

In $\S 4$ we use Corollary 3.4 to obtain a mod $C$ version of the BlakersMassey triad theorem in the nilpotent category, and in an appendix we discuss the modifications needed in our results if we only assume our fibration (0.1) to be quasi-nilpotent, that is, we no longer assume $\pi_{1} B$ nilpotent.

We adopt a notation based on that of [9] but avoiding (we hope!) the more idiosyncratic features of that notation. We regard the Serre class $C$ as fixed. Then if $\alpha: G \rightarrow G^{\prime}$ is a homomorphism of nilpotent groups (in particular, of abelian groups) we write $\iota G, \mu G, \varepsilon G$ to indicate that $\alpha$ is $C$-bijective, $C$-injective, $C$-surjective respectively; and we cross out the symbol $h \mu, \varepsilon$ to deny the assertion it represents. This notation enormously shortens our formulations; it is also appropriate since many of our assertions are proved by contradiction.

Unlike the statement of hypotheses in [9], which is purely algebraic, we state our hypotheses in topological guise in $\$ \S 1$ and 3 . However, it is quite obvious that these hypotheses can be rendered purely algebraic by anybody conscientious enough to undertake the task.

It is a pleasure to acknowledge the value of conversations with Guido Mislin. In particular, he drew our attention to Quillen's paper [6], in Lemma 3.8 of which he formulated 'the core of the Zeeman comparison theorem'. Quillen worked in cohomology and his hypotheses were inappropriate to our concern in this paper; nevertheless his formulation provided the clue as to how to generalize Zeeman's argument.

\section{Nilpotent local coefficients}

Let $X, X^{\prime}$ be connected spaces with nilpotent fundamental groups; let $C$ be an acyclic Serre class of nilpotent groups and suppose either that $C$ 
satisfies $(I I)$ or that $C$ satisfies $(I)$ and $X, X^{\prime}$ have finitely-generated homology groups; let $f: X \rightarrow X^{\prime}$ be a map inducing a $C$-bijection $\pi_{1} f: \pi_{1} X \rightarrow \pi_{1} X^{\prime}$; let $A, A^{\prime}$ be nilpotent $\pi_{1} X-, \pi_{1} X^{\prime}$-modules, respectively, and let $\phi: A \rightarrow A^{\prime}$ be a module-map compatible with $\pi_{1} f$. We prove three propositions relating to this situation.

Proposition 1.1. If $\iota A ; \iota H_{i} X, i<n$; and $\epsilon H_{n} X$, then $\iota H_{i}(X ; A), i<n$, and $\varepsilon H_{n}(X ; A)$.

Recall that $\iota A$ means that $\phi: A \rightarrow A^{\prime}$ is $C$-bijective; similarly $\varepsilon H_{n} X$ means that $H_{n} f: H_{n} X \rightarrow H_{n} X^{\prime}$ is $C$-surjective.

Proof. We argue by induction on $c=\max \left(\right.$ nil $_{\pi} A$, nil $\left._{\pi^{\prime}} A^{\prime}\right)$, where $\pi=$ $\pi_{1} X, \pi^{\prime}=\pi_{1} X^{\prime}$. If $c=1$, then the coefficients are trivial modules and the conclusion easily follows from the universal coefficient theorem in homology. For the inductive step, we write $\Gamma^{i}=\Gamma_{\pi}^{i} A, \Gamma^{i}=\Gamma_{\pi}^{i} A^{\prime}$, and consider the commutative diagram, with each vertical arrow $C$-bijective (Corollary 4.3 of $[4])$,

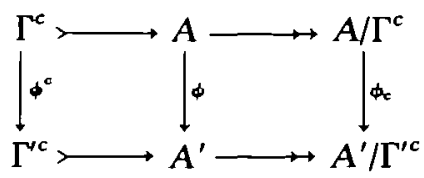

and the induced map of homology sequences

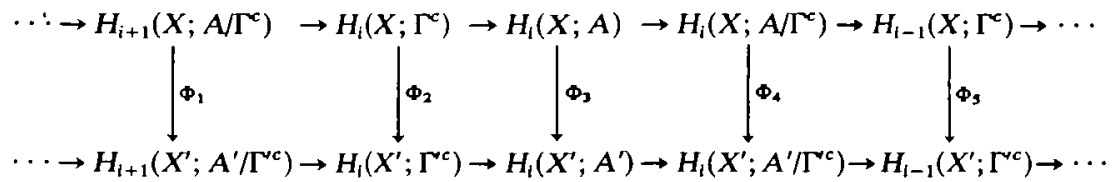

If $i<n$, then $\Phi_{1}$ is $C$-surjective, $\Phi_{2}, \Phi_{4}$ and $\Phi_{5}$ are $C$-bijective, all by the inductive hypothesis, so $\Phi_{3}$ is $C$-bijective. If $i=n$, then $\Phi_{2}, \Phi_{4}$ are $C$-surjective, $\Phi_{5}$ is $C$-bijective, all by the inductive hypothesis, so $\Phi_{3}$ is $C$-surjective.

Proposition 1.2. If $\iota H_{0}(X ; A), \varepsilon H_{1}(X ; A), \varepsilon H_{2} X$, then $\iota A$.

Proof. We prove by induction on $i$ that $\iota\left(A / \Gamma^{l}\right)$; the result will then follow by taking $i$ sufficiently large. Now $H_{0}(X ; A)=A / \Gamma^{2}=A_{\text {m }}$, so we have $\iota\left(A / \Gamma^{2}\right)$. If $\iota\left(A / \Gamma^{i}\right), i \geqslant 2$, consider the evident diagram

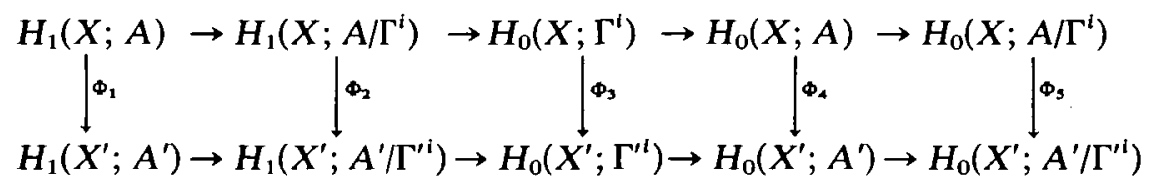


Now $\Phi_{1}$ is $C$-surjective and $\Phi_{4}$ is $C$-bijective by hypothesis. Since $\iota \pi_{1} X$ we have $\iota H_{1} X$ (Theorem 3.4 of [4]) so that, given $\varepsilon H_{2} X$ and the inductive hypothesis $\iota\left(A / \Gamma^{i}\right)$ we infer from Proposition 1.1 that $\Phi_{2}$ and $\Phi_{5}$ are $C$-bijective. It follows that $\Phi_{3}$ is $C$-bijective. But $H_{0}\left(X ; \Gamma^{l}\right)=\Gamma^{i} / \Gamma^{i+1}$ so that $\iota\left(\Gamma^{i} / \Gamma^{i+1}\right)$. We now conclude that $\iota\left(A / \Gamma^{i+1}\right)$ and the inductive step is complete.

Proposition 1.3. If $\varepsilon H_{0}(X ; A)$ then $\varepsilon A$.

Proof. We first factor $\phi_{2}: A / \Gamma^{2} \rightarrow A^{\prime} / \Gamma^{\prime 2}$ as

$$
A / \Gamma^{2} \stackrel{a}{\rightarrow} A^{\prime} / \Gamma_{\pi}^{2} A^{\prime} \stackrel{\beta}{\rightarrow} A^{\prime} / \Gamma^{2} .
$$

Here we construct $\Gamma_{\pi}^{2} A^{\prime}$ by regarding $A^{\prime}$ as a $\pi$-module via $\pi_{1} f$. Since $\iota \pi_{1} X$ we have $\iota H_{1} X$, so that $\beta$ is a $C$-bijection by Proposition 1.1 . Thus, since $\phi_{2}$ is $C$-surjective, so is $\alpha$, and we must deduce that $\phi$ is $C$ surjective. This argument allows us, in proving Proposition 1.3, to assume that $\pi=\pi^{\prime}$ and that $\pi_{1} f$ is the identity.

We now exploit the commutative diagram, with $I \pi$ the augmentation ideal of $\pi$,

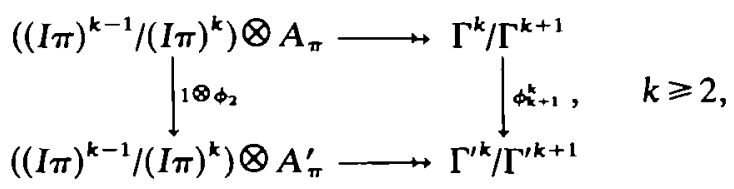

where the horizontal arrow is given by $[\xi] \otimes[a] \mapsto[\xi a], \xi \in(I \pi)^{k-1}, a \in A$. We note that $(I \pi)^{k-1} /(I \pi)^{k}=(I \pi)^{k-1} \bigotimes_{\pi} Z$ and that, if $H_{1} X$ is finitelygenerated, so is $\pi_{1} X$ and hence so is $(I \pi)^{k-1}$ as $\pi$-module. Thus on either hypothesis on the class $C$, we may infer that, $\phi_{2}$ being $C$-surjective, so is $1 \otimes \phi_{2}$ and hence also $\phi_{k+1}^{k}, k \geqslant 2$. Thus by the 5-lemma we may prove, by induction on $(j-k)$, that $\phi_{j}^{k}: \Gamma^{k} / \Gamma^{j} \rightarrow \Gamma^{\prime k} / \Gamma^{\prime j}$ is $C$-surjective, $2 \leqslant k<j$. Setting $k=2$ and taking $j$ sufficiently large we infer that $\phi^{2}: \Gamma^{2} \rightarrow \Gamma^{2}$ is $C$-surjective. Recourse to the diagram

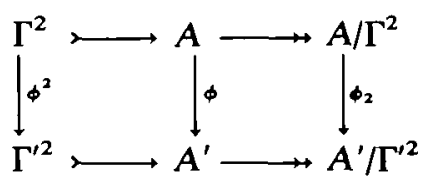

finally shows that $\phi$ is $C$-surjective.

We close this section by giving the explicit forms of these three propositions when $X, X^{\prime}$ are Eilenberg-MacLane spaces.

Corollary 1.4. If $\gamma: \pi \rightarrow \pi^{\prime}$ is a $C$-bijection; $A, A^{\prime}$ nilpotent $\pi$-, $\pi^{\prime}$-modules, respectively; $\phi: A \rightarrow A^{\prime}$ a module map compatible with $\gamma$, 
then

(i) if $\iota A$, then $\iota H_{i}(\pi ; A), i \geqslant 0$;

(ii) if $\iota H_{0}(\pi ; A), \varepsilon H_{1}(\pi ; A)$, then $\iota A$;

(iii) if $\varepsilon H_{0}(\pi ; A)$, then $\varepsilon A$.

Proof. It is only necessary to observe (Theorem 3.4 of [4]) that if $\gamma: \pi \rightarrow \pi^{\prime}$ is $C$-bijective, so is $H_{l} \gamma: H_{l} \pi \rightarrow H_{l} \pi^{\prime}, i \geqslant 0$.

\section{The Zeeman lemmas}

In this section we improve the crucial Lemma 3 and 4 of [9] in order to be able to prove a strengthened form of the comparison theorem. We emphasize that, in this section, we are not concerned with strengthening the comparison theorem in order to handle quasi-nilpotent fibrations, but in order to be able to weaken the assumptions of Theorem 2 and 3 of [9], even in the case of simply-connected bases. This weakening of the assumptions is already necessary in order to be able to obtain the Whitehead theorem as a consequence of the comparison theorem. We will also work modulo an acyclic Serre class $C$ instead of working absolutely, but this presents no additional difficulty.

Let $\alpha: A \rightarrow A^{\prime}$ be a homomorphism of abelian groups. Provided that it is evident that we are referring to $\alpha$, we will, as in Section 1 , write $\iota A$, $\mu A, \varepsilon A$ to mean that $\alpha$ is $C$-bijective, $C$-injective, $C$-surjective. By crossing out the symbol $\iota, \mu, \varepsilon$ we will deny the truth of the assertion conveyed by the symbol.

Now let $\alpha: K \rightarrow K^{\prime}$ be a homomorphism of finitely-filtered abelian groups. Let us write $F_{k}$ for the $k$ th term of either filtration. Then the following lemma improves Lemma 3 of [9].

LeMMA 2.1. (a) If $\mu K$ and $\mu F_{k} / F_{k-1}$, then $\varepsilon F_{j} / F_{j-1}$ for some $j<k$.

(b) If $\varepsilon K$ and $\ell F_{j} / F_{j-1}$, then $\alpha F_{k} / F_{k-1}$ for some $k>j$.

Proof. We will be content to prove $(b)$. We may assume, without loss of generality, that $F_{N} K=K, F_{N} K^{\prime}=K^{\prime}$. Let $k$ be the largest integer such that $\ell F_{k} / F_{k-1}$. Then $k \geqslant j$. Also it is easy to see by downward induction on $l$ and the 5-lemma that $\varepsilon F_{l}, k \leqslant l \leqslant N$. Thus $\varepsilon F_{k}$, whence $\varepsilon F_{k} / F_{k-1}$. 'It follows that $k \neq j$, so that $k>j$, and that $\mu F_{k} / F_{k-1}$.

To explain the second lemma, we need notation. Let us consider the first quadrant spectral sequence $E_{p q}^{r}, r \geqslant 2$, with deg $d^{r}=(-r, r-1)$ and let us set (see [9])

$$
\begin{gathered}
\langle p, q\rangle=\underset{\substack{p^{\prime}<p \\
q^{\prime}<q}}{\bigoplus} E_{p^{\prime} q^{\prime},}^{2}[n, p, s]=d^{p-s} E_{p, n-p}^{p-s}=B_{a, n-1-s,}^{p-1} 2 \leqslant s+2 \leqslant p \leqslant n, \\
{[n, p, \infty]=E_{p, n-p,}^{\infty} 0 \leqslant p \leqslant n .}
\end{gathered}
$$


Then if we consider $E_{p, n-p}^{2}, 2 \leqslant p \leqslant n-1$, we see that it is filtered by the subgroups, expressed by the customary abuse of language as

$0 \subseteq \operatorname{im} d^{2} \subseteq \operatorname{im} d^{3} \subseteq \cdots \subseteq \operatorname{im} d^{n-p} \subseteq \operatorname{im} d^{n+1-p}$

$$
\subseteq \operatorname{ker} d^{p} \subseteq \cdots \subseteq \operatorname{ker} d^{3} \subseteq \operatorname{ker} d^{2} \subseteq E_{p, n-p}^{2}
$$

and the associated graded group is given by

$$
\begin{aligned}
G r E_{p, n-p}^{2} & =[n+1, p+2, p]+[n+1, p+3, p]+\cdots \\
& +[n+1, n+1, p]+[n, p, \infty]+[n, p, 0]+\cdots+[n, p, p-2] .
\end{aligned}
$$

If $p=0,1$ or $n$ there is a simpler expression for the associated graded object; namely, if $p=0,1$, then we stop (2.2) at $[n, p, \infty]$ and, if $p=n$, we start $(2.2)$ at $[n, p, \infty]$. (We could simply adopt the convention that [n,p,s]=0 if $p=s+1$ or if $p=0,1$ and then (2.2) holds for $0 \leqslant p \leqslant n$.)

We write $\iota^{\prime}(p, q)$ to mean that $\iota E_{p^{\prime} q^{\prime}}^{2}$ if $p^{\prime}<q, q^{\prime} \leqslant q$ and $\varepsilon E_{p q^{\prime}}^{2}$ if $q^{\prime} \leqslant q$; and prove, improving Lemma 4 of [9],

\section{LemMa 2.2. If $\iota^{\prime}\langle p, n-1-s\rangle$ then $\iota[n, p, s]$.}

Proof. Suppose $\varepsilon[n, p, s]$. Since $\varepsilon E_{p, n-p}^{2}$, it follows from Lemma 2.1(b) and (2.2) that $\mu\left[n, p, s_{1}\right]$ for some $s<s_{1} \leqslant p-2$. Since $\iota E_{s_{1}, n-1-s_{1}}^{2}$ it follows from Lemma 2.1 (a) that $k\left[n, p_{1}, s_{1}\right]$ for some $s_{1}+2 \leqslant p_{1}<p$. Thus, iterating this argument, we find sequences

$$
s<s_{1}<s_{2}<\ldots, p>p_{1}>p_{2}>\cdots
$$

with $z\left[n, p_{i}, s_{i}\right]$. But this is absurd since we require $s_{i}+2 \leqslant p_{i}$.

The hypothesis $\mu[n, p, s]$ leads similarly to a contradiction.

\section{The comparison theorem for maps of fibre spaces}

We consider a map of fibrations, with all spaces connected,

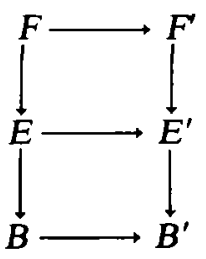

where it is assumed that $\pi_{1} B, \pi_{1} B^{\prime}$ are nilpotent and operate nilpotently on $H F, H F$ respectively. We take an arbitrary acyclic Serre class $C$ and we assume either that $C$ satisfies $(I I)$ or that $C$ satisfies $(I)$ and the homology groups of $F, F^{\prime}, B, B^{\prime}$ are finitely-generated. We prove first

TheOREM 3.1. If $\iota H_{q} F, q<Q, \varepsilon H_{Q} F$, and $\iota H_{p} B, p<P, \varepsilon H_{P} B, P \geqslant 2$, then $\iota H_{n} E, n<N=\min (P, Q), \varepsilon H_{N} E$. 
Proof. The hypotheses immediately imply (Theorem 3.4 of [4]) that $\iota \pi_{1} B$. It follows from Proposition 1.1 that $\iota E_{p q}^{2}$ for $p+q<N$ and $\varepsilon E_{p q}^{2}$ for $p+q=N$. It is then plain that we may pass through the spectral sequence to obtain

$$
\iota E_{p q}^{\infty} \text { for } p+q<N, \quad \varepsilon E_{p q}^{\infty} \text { for } p+q=N
$$

from which the theorem follows immediately.

We next prove

TheOREM 3.2. If $\iota H_{n} E, n<N, \varepsilon H_{N} E$; and $\iota H_{p} B, p<P, \varepsilon H_{P} B, P \geqslant 2$, then $\iota H_{q} F, q<Q=\min (N, P-1), \varepsilon H_{Q} F$.

We prove this as a consequence of the following more technical proposition.

Proposition 3.3. If $\iota H_{n} E, n<N, \varepsilon H_{N} E$; and $\iota H_{p} B, p<P, \varepsilon H_{P} B, P \geqslant 2$; and if $\iota H_{q} F, q<M$, then

$$
\begin{aligned}
& \text { if } M<Q, \iota E_{0 M}^{2} \text { and } \varepsilon E_{1 M}^{2} \\
& \text { if } M=Q, \varepsilon E_{0 M}^{2},
\end{aligned}
$$

where $Q=\min (N, P-1)$.

Deduction of Theorem 3.2 from Proposition 3.3.

Given the hypotheses of Theorem 3.2, then it follows that $\iota \pi_{1} B$ and that the hypotheses of Proposition 3.3 are certainly valid if $M=1$. If $Q>1$ we infer from Proposition 3.3 that $\iota E_{01}^{2}$ and $\varepsilon E_{11}^{2}$. But $\varepsilon H_{2} B$ by hypothesis so that, by Proposition 1.2, $\iota H_{1} F$. Similarly if $Q=1$ we infer from Proposition 3.3 that $\varepsilon E_{01}^{2}$ and hence, by Proposition 1.3, that $\varepsilon H_{1} F$. If $Q>1$ we may then take $M=2$. If $Q>2$ we infer, as above, that $\iota H_{2} F$ and if $Q=2$ we infer that $\varepsilon H_{2} F$. We continue in this way, finally obtaining $\iota H_{q} F, q<Q$, and $\varepsilon H_{Q} F$.

Proof of Proposition 3.3. From Proposition 1.1 we infer that $\iota^{\prime}\langle P, M-$ 1). We first suppose $M \leqslant Q$ and prove $\varepsilon E_{0 M}^{2}$. For, if $\varepsilon E_{0 M}^{2}$, then, by (2.2), either (i) $\ell[M, 0, \infty]$ or (ii) $\ell[M+1, p, 0]$ for some $2 \leqslant p \leqslant M+1$.

(i) If $\ell[M, 0, \infty]$ it follows from Lemma $2.1(b)$, since $\varepsilon H_{M} E$, that $\mu\left[M, p_{1}, \infty\right]$ for some $0<p_{1} \leqslant M$. But $p_{1} \neq M$, since $[M, M, \infty]$ is a subgroup of $H_{M} B$ and $\iota H_{M} B$. Thus $0<p_{1}<M$. Since $\iota E_{p_{1}, M-p_{1}}^{2}$, it follows from Lemma 2.1(a) that $E\left[M+1, p_{2}, p_{1}\right]$ for some $p_{1}+2 \leqslant p_{2} \leqslant M+1$. But $\imath^{\prime}\left\langle p_{2}, M-p_{1}\right\rangle$, contradicting Lemma 2.2 .

(ii) If $\varepsilon[M+1, p, 0]$ for some $2 \leqslant p \leqslant M+1$, then, since $\varepsilon E_{p, M+1-p}^{2}$ it follows from Lemma $2.1(b)$ that $\alpha[M+1, p, s]$ for some $1 \leqslant s \leqslant p-2$. But $\iota^{\prime}\langle p, M-s\rangle$, contradicting Lemma 2.2 .

We next prove that, if $M<Q$, then $\mu E_{0 M}^{2}$. For, if $\mu E_{O M}^{2}$ then, by (2.2), either (iii) $\alpha[M, 0, \infty]$ or (iv) $\mathcal{k}[M+1, p, 0]$ for some $2 \leqslant p \leqslant M+1$. 
(iii) Since $[M, 0, \infty]$ is a subgroup of $H_{M} E$ and $\iota H_{M} E$, it follows that $\mu[M, 0, \infty]$.

(iv) If $\boldsymbol{\mu}[M+1, p, 0]$ for some $2 \leqslant p \leqslant M+1$, then, since $\iota E_{p, M+1-p}^{2}$, it follows from Lemma $2.1(a)$ that $\varepsilon[M+1, p, \infty]$ or $\varepsilon\left[M+2, p_{1}, p\right]$ for some $p+2 \leqslant p_{1} \leqslant M+2$. The second possibility contradicts $\iota^{\prime}\left\langle p_{1}, M+1-\right.$ p). Thus $\varepsilon[M+1, p, \infty]$ whence, since $\varepsilon H_{M+1} E$, it follows from Lemma $2.1(b)$ that $\alpha\left[M+1, p_{1}, \infty\right]$ for some $p \leqslant p_{1} \leqslant M+1$. This possibility, however, is excluded exactly as in the argument (i).

Finally we prove that, if $M<Q$, then $\varepsilon E_{1 M}^{2}$. For, if $\varepsilon E_{1 M}^{2}$ then, by (2.2), either $(v) \varepsilon[M+1,1, \infty]$ or $(v i) \varepsilon[M+2, p, 1]$, for some $3 \leqslant p \leqslant M+2$.

(v) If $\varepsilon[M+1,1, \infty]$, it follows from Lemma $2.1(b)$, since $\varepsilon H_{M+1} E$, that $\boldsymbol{k}[M+1, p, \infty]$ for some $1<p \leqslant M+1$. We now argue as in (i).

(vi) If $\varepsilon[M+2, p, 1]$ for some $3 \leqslant p \leqslant M+2$ then, since $\varepsilon E_{p, M+2-p}^{2}$, it follows from Lemma $2.1(b)$ that $\alpha[M+2, p, s]$ for some $1<s \leqslant p-2$. But $\iota^{\prime}(p, M+1-s)$, contradicting Lemma 2.2 .

Corollary 3.4. Let $f: X \rightarrow X^{\prime}$ be a map of nilpotent spaces and let $C$ be an acyclic Serre class. Assume either that $C$ satisfies (II) or that $C$ satisfies $(I)$ and $X, X^{\prime}$ are of finite type. Then, if $n \geqslant 2$, the following statements are equivalent.

(i) $\iota H_{i} X$, i<n, and $\varepsilon H_{n} X$;

(ii) $\iota \pi_{i} X$, $i<n$, and $\varepsilon \pi_{n} X$;

(iii) $\iota \pi_{1} X, \iota H_{i} \tilde{X}, i<n$ and $\varepsilon H_{n} \tilde{X}$.

Proof. The equivalence of (ii) and (iii) is classical. To establish the equivalence of (i) and (iii) we first observe that $\iota H_{1} X, \varepsilon H_{2} X$ together imply $\iota \pi_{1} X$. We now consider the nilpotent fibrations

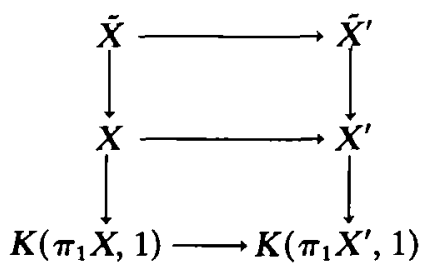

We next note that if $X, X^{\prime}$ are of finite type then the homology groups of all spaces in (3.2) are finitely generated. Thus we may, on the hypothesis (i) or (iii), apply Theorems $3.1,3.2$ with $P=\infty$. Then the implication (iii) $\Rightarrow$ (i) follows from Theorem 3.1 and the implication (i) $\Rightarrow$ (iii) follows from Theorem 3.2.

Remark. The equivalence of (i) and (ii), in the absolute case $C=\{1\}$, is known to Dror and has also been disovered independently by Toomer [8]. 
To complete the comparison theorem we revert to (3.1) and prove

THEOREM 3.5. If $\iota H_{n} E, n<N, \varepsilon H_{N} E$; and $\iota H_{q} F, q<Q, \varepsilon H_{Q} F$; and $\iota \pi_{1} B$, then $\iota H_{p} B, p<P=\min (N, Q+1), \varepsilon H_{P} B$.

Proof. We assume that $\imath H_{p} B, p<M, \varepsilon H_{M} B$ and show that $\imath H_{M} B$, $\varepsilon H_{M+1} B$, provided that $M<P$. This will prove the theorem since certainly we may begin with $M=1$. From our assumption we infer that $\iota^{\prime}\langle M, Q-1\rangle$. We first prove that $\mu H_{M} B$. If $\alpha E_{M 0}^{2}$, then either $(i)$ $\alpha[M, M, \infty]$ or (ii) $\alpha[M, M, p]$ for some $0 \leqslant p \leqslant M-2$.

(i) If $\alpha[M, M, \infty]$ then, since $\iota H_{M} E, \ell\left[M, p_{1}, \infty\right]$ for some $0 \leqslant p_{1}<M$. But $p_{1} \neq 0$ since $[M, 0, \infty]$ is a quotient of $H_{M} F$ and $\varepsilon H_{M} F$. Thus $0<p_{1}<$ $M$. Now $\iota E_{p_{1}, M-p_{1}}^{2}$ so $\mu\left[M, p_{1}, s\right]$ for some $0 \leqslant s \leqslant p_{1}-2$. But this contradicts $\iota\left\langle p_{1}, M-1-s\right\rangle$.

(ii) If $\alpha[M, M, p]$ then, since $\ell E_{p, M-1-p}^{2}$, it follows that $\ell\left[M, p_{2}, p\right]$ for some $p+2 \leqslant p_{2}<M$. But this contradicts $\iota\left\langle p_{2}, M-1-p\right\rangle$.

We next prove that $\varepsilon H_{M+1} B$. If $\varepsilon E_{M+1,0}^{2}$, then either (iii) $\varepsilon[M+1, M+$ $1, \infty]$ or (iv) $\ell[M+1, M+1, p]$ for some $0 \leqslant p \leqslant M-1$.

(iii) Since $\varepsilon H_{M+1} E$ it follows that $\varepsilon[M+1, m+1, \infty]$.

(iv) If $\varepsilon[M+1, M+1, p]$ then, since $\iota E_{\mathrm{p}, M-p}^{2}$ if $p>0$ and $\varepsilon E_{0 M}^{2}$ (for $E_{0 M}^{2}$ is a quotient of $H_{M} F$ and $\left.\varepsilon H_{M} F\right)$, it follows that $\alpha[M, p, \infty]$ or $\mu[M, p, s]$ for some $0 \leqslant s \leqslant p-2$. The second possibility is excluded since $\iota\langle p, M-1-s\rangle$. The first possibility implies, since $\iota H_{M} E$, that $\ell\left[M, p_{1}, \infty\right]$ for some $0 \leqslant p_{1}<p$ and is excluded exactly as in (i).

\section{A Blakers-Massey triad theorem in nilpotent $C$-theory}

In the authors' earlier paper [4], use was made of the implication (ii) $\Rightarrow(i)$ in Corollary 3.4 to study torsion phenomena in nilpotent spaces. However, in classical homotopy theory, it is often the converse implication (i) $\Rightarrow$ (ii) which plays a crucial role. In this section, we use this more delicate half of our extended Whitehead theorem to similarly extend the Blakers-Massey triad theorem.

A convenient source for the mod C Blakers-Massey triad theorem in the classical setting is ([1]; Paper 7) and we shall be content to show how to modify the proof contained therein so as to obtain the following more general statement:

THEOREM 4.4. Suppose that $(X ; A, B)$ is a $C W$-triad, that each of the spaces $X, A, B, C=A \cap B$ is (connected) nilpotent and that the inclusion $C \subseteq X$ induces a surjection of fundamental groups. Suppose further that $\pi_{r}(X, A) \in S, r<q, q \geqslant 3$ and $\pi_{r}(X, B) \in S, r<p, p \geqslant 3$, where $S$ is an acyclic Serre class; either $S$ satisfies (II) or $S$ satisfies $(I)$ and $X, A, B, C$ are of finite type. Then the triad homotopy groups $\pi_{r}(X ; A, B) \in S, r<$ 
$p+q-1$ and the generalized Whitehead product $\pi_{p}(A, C) \otimes \pi_{q}(B, C) \rightarrow$ $\pi_{\mathrm{p}+\mathrm{q}-1}(X ; A, B)$ is a $S$-bijection.

Proof. We shall, as indicated in the preamble, restrict ourselves to commenting on the various places in the proof given in [1] where modification is necessary.

In Step $1,([1] ;$ p. 109$)$, the triad $(X ; A, B)$ is replaced by a triad $\left(X_{1} ; A_{1}, B_{1}\right)$; here $X_{1}=(X, \text { basepoint })^{(0,1], 0)}$ is the (contractible) path space, $A_{1}=(X, A)^{[0,1], 0)}, B_{1}=(X, B)^{[0,1], 0)}$ and $C_{1}=(X, C)^{[0,1], 0)}=A_{1} \cap$ $B_{1}$. Clearly, the relative and triad homotopy groups of $\left(X_{1} ; A_{1}, B_{1}\right)$ are the 'same' as those of $(X ; A, B)$, and $\left(X_{1} ; A_{1}, B_{1}\right)$ is a $C W$-triad by the result of Milnor. It is only necessary to check that each of the spaces $X_{1}$, $A_{1}, B_{1}, C_{1}$ is (connected) nilpotent. For $A_{1}$, for example, we have the fibration (up to homotopy)

$$
A_{1} \rightarrow A \rightarrow X,
$$

where $A \rightarrow X$ is the inclusion. By assumption, $\pi_{1}(C) \rightarrow \pi_{1}(X)$, hence also $\pi_{1}(A) \rightarrow \pi_{1}(X)$, so that $A_{1}$ is connected. It then follows from the fact that $A$ is nilpotent, using [2] (or [3]) that $A_{1}$ is also nilpotent.

In Step 2, ([1]; pp. 109, 110), the triad $\left(X_{1}, A_{1}, B_{1}\right)$ is replaced by a triad $\left(X_{2} ; A_{2}, B_{2}\right)$; here $X_{2}=X_{1} \times X_{1}, A_{2}=A_{1} \times B_{1}, B_{2}=$ diagonal in $X_{2}$ and $C_{2}=A_{2} \cap B_{2}$ is homeomorphic to $C_{1}$. Again, the relative and triad homotopy groups of $\left(X_{2} ; A_{2}, B_{2}\right)$ are the 'same' as those of $\left(X_{1} ; A_{1}, B_{1}\right)$, but to make $\left(X_{2} ; A_{2}, B_{2}\right)$ a $C W$-triad it may be necessary to retopologize. Plainly, the spaces $X_{2}, A_{2}, B_{2}, C_{2}$ are nilpotent since $X_{1}, A_{1}, B_{1}, C_{1}$ are nilpotent.

Finally, in Step 3, ([1]; p. 111, 112), we use Corollary 3.4 to infer from the assertion $H_{r}\left(A_{2}, C_{2}\right) \in S, r<p+q-2$, that also $\pi_{r}\left(A_{2}, C_{2}\right) \in S, r<$ $p+q-2$. In fact, the hypotheses $p \geqslant 3, q \geqslant 3$, needed elsewhere in the proof, imply a fortiori that $p+q-2>2$, thus insuring fulfillment of the dimensionality criterion in Corollary 3.4. (The hypotheses $p \geqslant 3, q \geqslant 3$ are needed only to establish the last clause of the theorem, involving the generalized Whitehead product. The 'vanishing' assertion $\pi_{r}(X ; A, B) \in$ $S, r<p+q-1$, only requires $p+q \geqslant 5$.) Furthermore, it is evident that one deduces the finite type of $X_{2}, A_{2}, B_{2}, C_{2}$ from that of $X, A, B, C$ so that Corollary $3.4((i) \Rightarrow(i i))$ may indeed be applied.

The rest of the argument in [1] is unchanged.

\section{Appendix}

So far as the theorems of $\S 3$ are concerned, we only needed the assumption that the bases of our fibrations have nilpotent fundamental groups, in order to be able to apply nilpotent $C$-theory [4]. If we are 
prepared to assume that, in the map of fibrations (3.1), the induced map $\pi_{1} B \rightarrow \pi_{1} B^{\prime}$ is an isomorphism, then we may dispense with the condition that $\pi_{1} B, \pi_{1} B^{\prime}$ be nilpotent. In particular, in the absolute case $(C=\{1\})$ we will have no need of this hypothesis.

The precise modifications needed in our statements in Sections 1 and 3 are as follows; note that we now only need talk of a Serre class of abelian groups.

Propositions 1.1, 1.2, 1.3. We no longer assume $\pi_{1} X, \pi_{1} X^{\prime}$ nilpotent, but now assume $\pi_{1} f$ to be an isomorphism: the propositions then read exactly as stated, except that we must assume $\pi_{1} X$ finitely-generated if $C$ satisfies (I).

Corollary 1.4. We modify the hypothesis by assuming that $\gamma$ is an isomorphism with $\pi, \pi^{\prime}$ arbitrary groups; we must further assume that $\pi$ and $H_{i} \pi$ are finitely-generated if $C$ satisfies $(I)$. The conclusion of the corollary now follows.

Theorems 3.1, 3.2, 3.5. We no longer assume $\pi_{1} B, \pi_{1} B^{\prime}$ nilpotent, but assume $\pi_{1} B \rightarrow \pi_{1} B^{\prime}$ to be an isomorphism; the theorems then read exactly as stated. Note, however, that, with regard to Theorems 3.1, 3.2, there is a substantial price to be paid for dropping the requirement that $\pi_{1} B, \pi_{1} B^{\prime}$ be nilpotent. For, under that hypothesis, we could actually infer, from the rest of the data of the theorems, that $\pi_{1} B \rightarrow \pi_{1} B^{\prime}$ was a C-bijection.

\section{REFERENCES}

1. J. F. Adams, 'Algebraic Topology-A Student's Guide', Cambridge University Press (1972).

2. A. K. Bousfield and D. M. Kan, 'Homotopy limits, completions and localizations', Lecture Notes in Mathematics, 304, Springer (1972).

3. P. Hilton, G. Mislin and J. Roiberg, 'Localization of nilpotent groups and spaces', Notas de matemática, North-Holland Mathematics Studies 15 (1975).

4. P. Hilton and J. Roitberg, 'Generalized $C$-theory and torsion phenomena in nilpotent spaces' Houston Joum of Math. (to appear).

5. J. C. Moore, 'Algèbres d'Eilenberg-MacLane et homotopie', Séminaire de H. Cartan de l'E.N.S. vii exposé ii (Paris, 1954-5).

6. D. Quillen, 'An application of simplicial profinite groups', Comm. Math. Helv. 44 (1969), 45-61.

7. J.-P. Serre, 'Groupes d'homotopie et classes de groupes abéliens', Ann. of Math 58 (1953), 258-294.

8. G. H. Toomer, 'The relative Hurewicz theorem for nilpotent spaces' (preprint)

9. E. C. Zeeman, 'A proof of the comparison theorem for spectral sequences', Proc. Cam. Phil. Soc. 53 (1957), 57-62.

Battelle Seattle Research Center, Case Western Reserve University, Cleveland, and Eidgenössische Technische Hochschule, Zürich Hunter College and Graduate Center of CUNY, New York 